\title{
Effects of Dietary Serine Supplementation on Intestinal Integrity, Inflammation and Oxidative Status in Early-Weaned Piglets
}

\author{
Xihong Zhou ${ }^{a, c} \quad$ Yumei Zhang ${ }^{a, b} \quad$ Xin Wu ${ }^{a, b} \quad$ Dan Wan $^{a} \quad$ Yulong Yin ${ }^{a, b}$ \\ aKey Laboratory of Agro-ecological Processes in Subtropical Region, Institute of Subtropical \\ Agriculture, Chinese Academy of Sciences, Hunan Provincial Key Laboratory of Animal Nutritional \\ Physiology and Metabolic Process, Hunan Provincial Engineering Research Center for Healthy Livestock \\ and Poultry Production; Scientific Observing and Experimental Station of Animal Nutrition and Feed \\ Science in South-Central, Ministry of Agrictulture, Changsha, 'bunan Co-Innovation Center of Animal \\ Production Safety, CICAPS, College of Animal Science and Technology, Hunan Agricultural University, \\ Changsha, 'State Key Laboratory of Animal Nutrition, China Agricultural University, Beijing, China
}

\section{Key Words}

Inflammation • Intestine integrity $\bullet$ Oxidative stress $\bullet$ Serine $\bullet$ Weaning

\begin{abstract}
Background/Aims: Early weaning often causes gut dysfunction. Since serine alleviates oxidative stress and inflammatory response which are accompany with early weaning, we conducted the study to explore whether serine improves intestinal function in early-weaned piglets. Methods: Twenty-eight weaned piglets (aged $21 \mathrm{~d}$ ) were fed either a basal diet or a basal diet plus $0.2 \%$ serine. We determined the effects of dietary serine supplementation on intestinal morphology by hematoxylin and eosin staining, expression of tight junction proteins (TJPs) by immunoblotting and immunofluorescence, expression of inflammatory cytokines and apoptosis markers by RT-qPCR and the level of antioxidant enzymes with ELISA kits in early-weaned piglets. Results: Serine supplementation increased daily body weight gain while decreasing diarrhea incidence. Both the jejunum and ileum of serine-supplemented piglets showed regularly arranged villi and microvilli. Moreover, dietary serine increased TJP expression, and alleviated apoptosis, inflammation, and oxidative stress in the intestine of early-weaned piglets. Conclusion: Our findings suggest that serine has the potential for use as a feed additive to prevent gut dysfunction caused by weaning.
\end{abstract}




\section{Cellular Physiology Cell Physiol Biochem 2018;48:993-1002

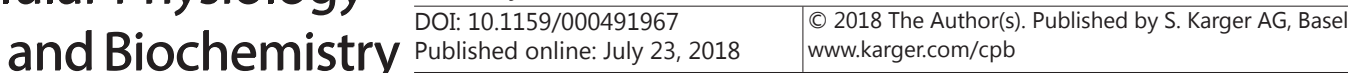 \\ Zhou et al.: Serine Protects Intestinal Function in Piglet}

\section{Introduction}

Nutritional, social and environmental factors such as changes in diet, new littermates, and pathogens induce strong stresses at weaning in piglets [1]. These stressors cause loss of appetite, growth retardation, high incidence of diarrhea, and increased risks of diseases and mortality [2]. These performances were often caused by intestinal dysfunction, which is characterized by morphological damage, tight junction injury, inflammation, apoptosis and oxidative stress $[1,3]$. Many strategies have been used to alleviate oxidative stress, maintain gut health and improve growth performance of early-weaned piglets. For decades, antibiotics have been the commonly used remedy. However, development of bacterial cross-resistance due to overuse of antibiotics has led to restrictions in many countries. Therefore, studies are necessary for identifying alternative non-antibacterial growth promoters.

Serine, recently classified as a conditionally non-essential amino acid, has been suggested as playing important roles ranging from protein synthesis to cell signal transduction, the latter mostly through post-translational modification by phosphorylation $[4,5]$. Research examining the impacts of dietary serine supplementation on humans and rodents showed no adverse effects [4]. Our recent studies with mice showed that serine, as a precursor of glutathione, could alleviate hepatic oxidative stress by regulating the expression of glutathionesynthesis-related genes and increasing glutathione concentration [6, 7]. In addition, serine supplementation has been reported to be beneficial for intestinal health in a mouse model with intestinal inflammation and barrier damage induced by lipopolysaccharide (LPS) [8]. However, to our knowledge, no previous studies have considered the effects of dietary serine supplementation on piglets. Therefore, the present study investigated the effects of dietary serine supplementation on intestinal integrity, and inflammatory and oxidative status in early-weaned piglets.

\section{Materials and Methods}

\section{Animal and Experimental Design}

The experimental protocol was approved by the Protocol Management and Review Committee of Institute of Subtropical Agriculture, Chinese Academy of Science and Pigs were cared for and slaughtered according to the guidelines of Institute of Subtropical Agriculture on Animal Care (Changsha, China). Twenty-eight piglets with a mean body weight (BW) of $6.5 \pm 0.19 \mathrm{~kg}$ were weaned at the age of 21 days, and randomly assigned to two treatments (7 pens per treatment and 2 piglets per pen). Piglet in one treatment were fed a basal diet (Control group) (Table 1) and those in the other treatment were fed the basal diet plus $0.2 \%$ L-serine (Aladdin, Shanghai, China) (Serine group). This level of L-serine was chosen because our preliminary experiment showed that other levels of L-serine supplementation $(0.05 \%, 0.1 \%$ and $0.5 \%$ ) had no significant effects on growth performance in piglets. The composition and nutrient levels of the basal diet met recommended nutrient requirements of NRC (2012). During the experiment, piglets were fed the relevant diet three times per day at 08:00, 13:00, and 18:00, and were given free access to water. The duration of the whole experiment was 4 weeks.

\section{Growth Performance and Sample Collection}

Feed intake was recorded every day and body weight was recorded every 2 weeks. Average daily weight gain and feed intake were calculated. The severity of scours ( 1 = hard feces; 2 = no scours, feces of normal consistency; 3 = mild scours, soft, partially formed feces; 4 = moderate scours, loose, semi-liquid feces; 5 = watery feces;) based on Sweet et al. (1990) [9] were scored blind on a daily basis by an investigator unaware of the piglet diets used in the study. At the end of the experiment, the average diarrhea score (total score during the

Table 1. Composition of the basal diet

\begin{tabular}{lc}
\hline Ingredient & $\%$ \\
\hline Corn & 61.5 \\
Soybean meal & 22 \\
Soybean oil & 2 \\
Fish meal & 4 \\
Whey powder & 7 \\
NaCl & 0.3 \\
Zinc oxide & 0.3 \\
Calcium hydrophosphate & 0.5 \\
Mountain flour & 0.9 \\
Choline chloride (50\%) & 0.06 \\
Lysine (78\%) & 0.5 \\
Methionine & 0.1 \\
Mineral & 0.03 \\
Vitamin & 0.01 \\
Medical stone & 0.8 \\
\hline
\end{tabular}




\section{Cellular Physiology Cell Physiol Biochem 2018;48:993-1002 and Biochemistry Published online: July 23, $2018 \quad \begin{aligned} & \text { DOI: 10.1159/000491967 } 2018 \text { The Author(s). Published by S. Karger AG, Basel } \\ & \text { www.karger.com/cpb }\end{aligned}$ \\ Zhou et al.: Serine Protects Intestinal Function in Piglet}

experiment/28 days) for each pen was calculated. At 09:00 on day 29, after overnight fasting, blood samples were taken from the jugular vein of the piglets. They were then anesthetized with sodium pentobarbital intravenously (50 mg/kg BW) followed by exsanguination. The liver, pancreas, and kidney of each piglet were weighed, and tissue samples from the jejunum and ileum were collected (after being cleaned with ice-cold phosphate-buffered saline), frozen immediately in liquid nitrogen, and stored at $-80{ }^{\circ} \mathrm{C}$. In addition, one jejunal and ileal segment were either fixed in $10 \%$ neutral buffered formalin or $2.5 \%$ glutaraldehyde or embedded in OCT embedding medium for further analysis.

\section{Inflammatory Cytokines Determination}

Inflammatory cytokines in serum were determined with ELISA kits (Cusabio, Wuhan, China) according to the manufacturer's instructions.

\section{Intestinal Histomorphology}

Jejunal and ileal samples fixed with 4\% formaldehyde, were embedded in paraffin, and sections, $8 \mu \mathrm{m}$ in thickness, were obtained and stained with hematoxylin and eosin (HE). Villus length and crypt depth were measured as in a previous study [10]. In brief, villus height was defined as the distance from the villus tip to the crypt mouth, and crypt depth from the crypt mouth to the base. Jejunal and ileal samples fixed with $2.5 \%$ glutaraldehyde were fixed with osmium tetroxide and dehydrated with graded alcohol. The samples were then embedded in Epon-Araldite resin and ultrathin sections obtained. The sections were stained with lead citrate and uranyl acetate, and their images were taken with a Zeiss 902 transmission electron microscope (TEM).

Superoxide Dismutase, Glutathione Peroxidase, Malondialdehyde and Catalase Determination

Superoxide dismutase (SOD), glutathione peroxidase (GSH-Px), malondialdehyde (MDA) and catalase (CAT) in jejunal and ileal samples were determined with ELISA kits (Northwest Life Science Specialties, Vancouver, WA, USA) according to the manufacturer's instructions.

\section{Real-time Quantitative RT-PCR}

Total RNA extracted from jejunal and ileal samples was reverse-transcribed into cDNA using reverse transcriptase (Takara, Tokyo, Japan). The sequences of Primers (5'-3') used for PCR were presented in Table 2. The reaction was performed with a total of $10 \mu \mathrm{L}$ of assay solution containing $3 \mu \mathrm{L}$ deionized water, $5 \mu \mathrm{L}$ SYBR Green mix (Takara), $0.2 \mu \mathrm{L}$ Rox, $1 \mu \mathrm{L}$ cDNA template, and $0.4 \mu \mathrm{L}$ each of forward and reverse primers. Relative gene expression was expressed as a ratio of the target gene to the control gene using the formula $2^{-(\Delta \Delta \mathrm{CT})}$, where $\Delta \Delta \mathrm{CT}=\left(\mathrm{CT}_{\text {Target }}-\mathrm{Ct}_{\mathrm{GAPDH}}\right)_{\text {treatment }}-\left(\mathrm{CT}_{\text {Target }}-\mathrm{CT}_{\mathrm{GAPDH}}\right)_{\text {control }}$ as in a previous study [11].

Terminal deoxynucleotidyl transferase (TdT)-mediated biotin-16-dUTP nick end labeling (TUNEL) staining

Apoptosis was detected by TUNEL staining of sections of jejunal and ileal tissue using an in situ cell death detection kit (Roche, Shanghai, China) according to the manufacturer's instructions.

\section{Western blotting}

Total protein $(20 \mu \mathrm{g}$ per lane) extracted from jejunal and ileal samples was separated by SDS-PAGE and blotted onto PVDF membranes. Primary antibodies against nuclear

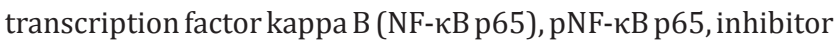
of nuclear factor kappa-B kinase-alpha $(\mathrm{I} \kappa \mathrm{B} \alpha)$, claudin-1 (Cell Signaling, Beverly, MA, USA), ZO-1 (Proteintech, Rosemont, IL, USA), occludin (Abcam, Shanghai, China), and $\beta$-actin (Boster, Wuhan, Hubei, China) were incubated with the membranes overnight at $4{ }^{\circ} \mathrm{C}$. After incubation overnight with secondary antibodies, the membranes were exposed to EZ-ECL (Biological Industries, Cromwell, CT, USA) to detect protein bands.

Table 2. Primer sequences for RT-qPCR

\begin{tabular}{lc}
\hline Gene & 5'-3' Primer sequence \\
\hline \multirow{2}{*}{ TNF- $\alpha$} & F: CCACGTTGTAGCCAATGTCA \\
& R: CAGCAAAGTCCAGATAGTCG \\
IL-1 $\beta$ & F: TGCCACCTTTTGACAGTGATG \\
& R: TCTTCATCGGCTTCTCCACT \\
IL-6 & F: CAAAGCCACCACCCCTAAC \\
& R: TCGTTCTGTGACTGCAGCTT \\
IL-8 & F: AGAACTGAGAAGCAACAACAACAG \\
& R: CACAGGAATGAGGCATAGATGTAG \\
Bcl-2L1 & F: TGAATCAGAAGCGGAAACCC \\
& R: GCTCTAGGTGGTCATTCAGGTAAG \\
cFLIP & F: GAGCAAGCCCCTAGGAATCT \\
& R: GTCTTGGTGTTGGGGCATAC \\
Caspase 3 & F: ACACGCCATGTCATCTTCAGTCC \\
& R: TTCATAATTCAGGCCTGCCGAG \\
Bax & F: AAGCGCATTGGAGATGAACT \\
& R: CGATCTCGAAGGAAGTCCAG \\
$\beta$-actin & F: CTGCGGCATCCACGAAACT \\
& R: AGGGCCGTGATCTCCTTCTG \\
\hline
\end{tabular}




\section{Cellular Physiology Cell Physiol Biochem 2018;48:993-1002 \begin{tabular}{c|c} 
DOI: 10.1159/000491967 & $\begin{array}{l}\text { O 2018 The Author(s). Published by S. Karger AG, Basel } \\
\text { www.karger.com/cpb }\end{array}$
\end{tabular} \\ Zhou et al.: Serine Protects Intestinal Function in Piglet}

Immunofluorescence Assay

Sections $(8 \mu \mathrm{m})$ were stained with one of three primary antibodies (claudin-1, occluding, and ZO-1) overnight at $4{ }^{\circ} \mathrm{C}$. After incubation with the appropriate secondary antibodies at $37^{\circ} \mathrm{C}$ for $1 \mathrm{~h}$, each section was mounted on a slide with SlowFade Gold antifade reagent containing 4',6'-diamidino-2-phenylindole (DAPI) (Sigma, Shanghai, China) and observed under a fluorescent microscope.

\section{Statistical Analysis}

Data were analyzed using the independent samples t-test of SPSS 17.0 (SPSS Inc., Chicago, IL, USA). Values are presented as means with standard errors, and mean values were considered to be significantly different when $\mathrm{P}<0.05$.

\section{Results}

\section{Growth performance}

We first observed the effects of serine supplementation on growth performance. Fig. 1 shows that there were no significant differences between piglets in the Control group and piglets in the Serine group in initial body weight (Fig 1A), daily feed intake (Fig 1D), feed to gain ratio (Fig 1E), and weight of the liver (Fig 1G), pancreas (Fig 1H), and kidney (Fig 1I). Final body weight and average daily weight gain were higher (Fig 1B, 1C) whereas diarrhea score was significantly lower (Fig 1F) in the Serine group than in the Control group.

\section{Intestinal histomorphology}

To explore the effects of serine supplementation on intestinal histomorphology, we observed intestinal villi and microvilli. According to the HE staining results, both jejunal and ileal villi of pigs in the Control group underwent unusual changes such as partial loss of shape and sloughing (Fig 2E, 2G). In contrast, the Serine group did not show the above changes (Fig 2F, 2H). Villus height was significantly greater (Fig 2A, 2C) in the Serine group than in the Control group, whereas no significant difference was observed in crypt depth (Fig 2B, 2D). Moreover, both the jejunum and ileum of pigs in the Serine group contained much more regularly aligned and extensive microvilli according to the TEM results (Fig 2I-2L).

\section{Expression of tight junction proteins}

To further explore the effects of serine supplementation on intestinal function, expression of tight junction proteins in the jejunum and ileum were detected and the results are shown in Fig. 3. Expression of claudin-1, occludin, and ZO-1 proteins was significantly higher in the Serine group than in the Control group according to the results of the immunofluorescence assay (Fig 3A-3L) and western blotting (Fig 3M-30).

\section{Apoptosis in the jejunum and ileum}

To explore whether intestinal histomorphology impairment is association with apoptosis, the level of apoptosis in the jejunum and ileum were observed and the results are shown in Fig. 4. The level of apoptosis in Control piglets was significantly higher than that in piglets supplemented with serine (Fig 4A-4D). Moreover, mRNA expression of anti-apoptotic genes (cFLIP and Bcl-2L1) (Fig 4G-4H) was significantly higher whereas mRNA expression of pro-apoptotic genes (caspase 3 and Bax) (Fig 4E-4F) was significantly lower in piglets supplemented with serine than in Control piglets.

\section{Oxidative status}

To explore whether intestinal dysfunction is association with oxidative stress, several antioxidants and lipid oxidation marker were detected. Fig. 5 shows that SOD, GSH-Px, and CAT concentrations (Fig 5A-5C) were significantly higher, whereas MDA concentration (Fig 5D) was significantly lower in both the jejunum and ileum of piglets in the Serine group than of those in the Control group. 


\section{Inflammatory cytokines}

To explore whether intestinal dysfunction is association with inflammatory response, inflammatory cytokines were detected. Fig. 6 shows that serum concentrations of TNF- $\alpha$, IL-1B, IL-6 and IL-8 (Fig 6A-6D), as well as their mRNA expression (Fig 6E-6H), in both the jejunum and ileum of piglets in the Control group were significantly higher than those of piglets in the Serine group. Relative protein expression of phosphorylated NF- $\kappa B$ and IкB was also significantly higher in the former than in the latter (Fig 6I-6K).

Fig. 1. Effects of serine on growth performance, diarrhea score and organ weight in early-weaned piglets. Control, piglets fed the basal diet; Serine, piglets fed the basal diet supplemented with $0.2 \%$ serine. Values are expressed as mean \pm SEM, $n$ $=7{ }^{*} \mathrm{p}<0.05$.
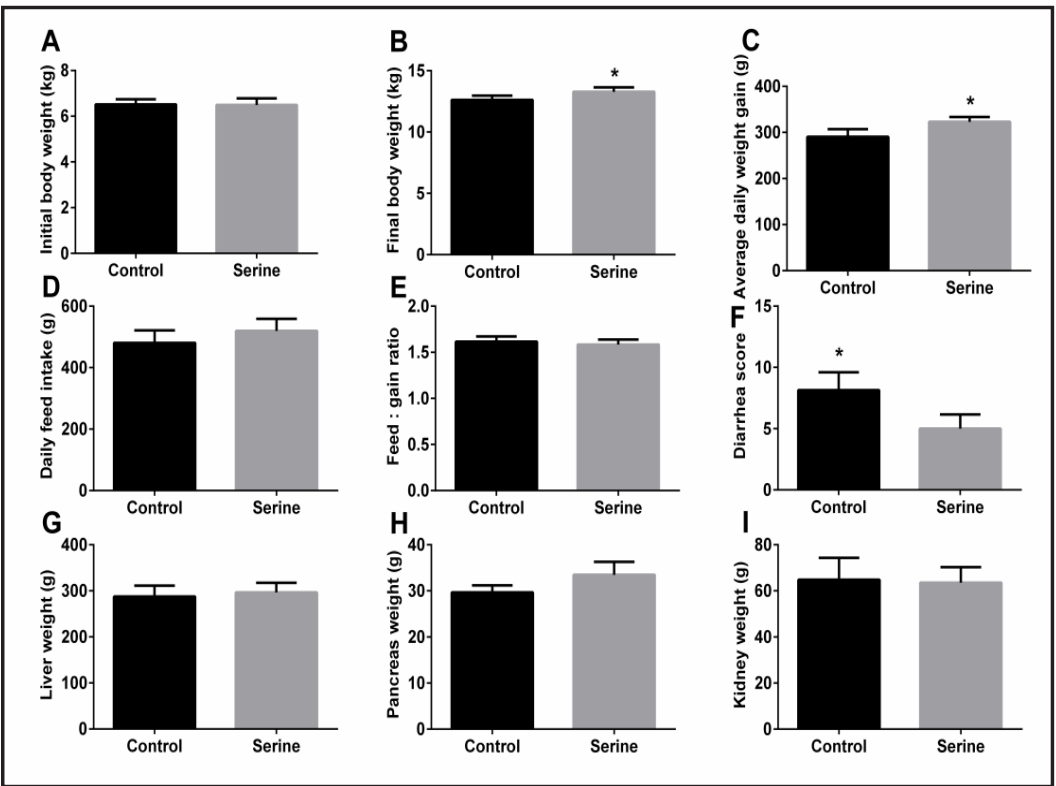

Fig. 2. Effects of serine on intestinal histomorphology in early-weaned piglets. A- B, villus height and crypt depth in jejunum; C-D, villus height and crypt depth in ileum; $\mathrm{E}-\mathrm{H}, \mathrm{HE}$ staining of intestinal morphology $(100 \times)$; Arrows, partial loss of shape and sloughing of villi (E, jejunum in control piglets; $F$, jejunum in serine-supplemented piglets; G, ileum in control piglets; $\mathrm{H}$, ileum in serine-supplemented piglets); I-L, ultrastructural
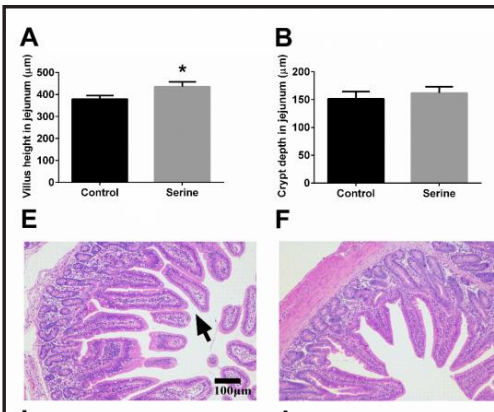

F
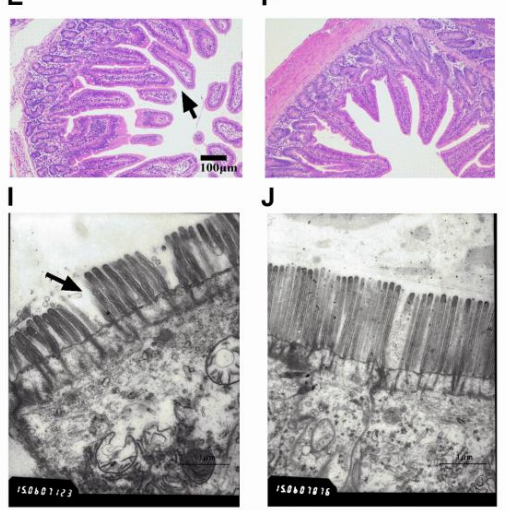

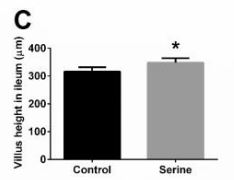

G

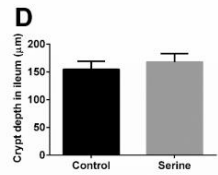

H

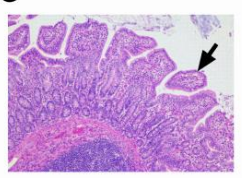

K

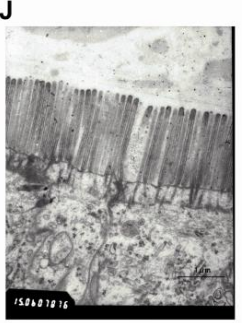

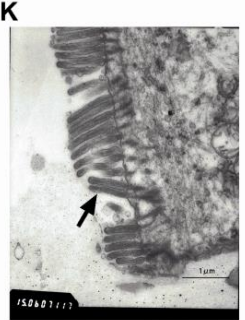

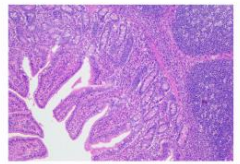

L

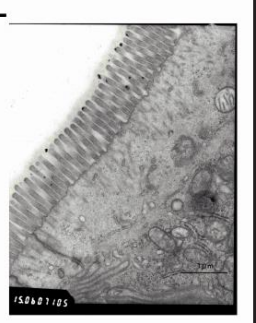
observation of microvilli

in ileum; Arrows, irregularly arranged microvilli (transmission electron microscopy, 15, 000×) (I, jejunum in control piglets; J, jejunum in serine-supplemented piglets; $\mathrm{K}$, ileum in control piglets; L, ileum in serinesupplemented piglets). Values are expressed as mean $\pm \mathrm{SEM}, \mathrm{n}=7$; $^{*} \mathrm{p}<0.05$. 


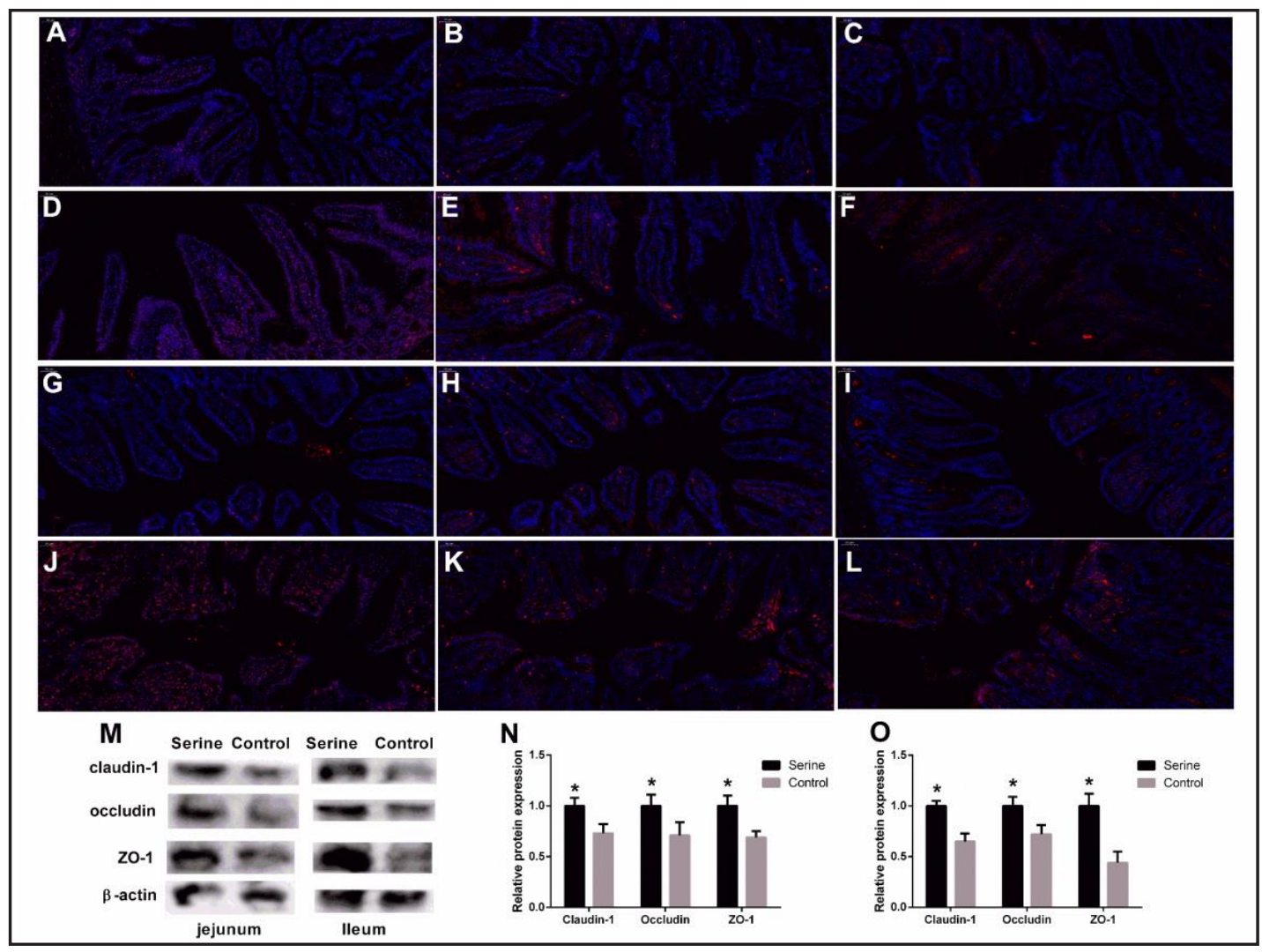

Fig. 3. Effects of serine on intestinal expression of tight junction proteins in early-weaned piglets. A-L, Immunofluorescence assay (80×) (A-C, claudin-1, occludin and Z0-1 expression in jejunum of control piglets; D-E, claudin-1, occludin and ZO-1 expression in jejunum of serine-supplemented piglets; G-I, claudin-1, occludin and ZO-1 expression in ileum of control piglets; J-L, claudin-1, occludin and ZO-1 expression in ileum of serine-supplemented piglets); $\mathrm{M}$, Western blot analysis of protein expression; $\mathrm{N}-\mathrm{O}$, protein expression of claudin-1, occludin and ZO-1 in jejunum and ileum. Red, tight junction proteins; Blue, nuclei stained with DAPI. Values are expressed as mean \pm SEM, $n=7 ;{ }^{*} p<0.05$.

Fig. 4. Effects of serine on levels of intestinal apoptosis in early-weaned piglets. A-D, Representative TUNEL (green) staining of ileum (200x) (A-B, results of jejunum in control piglets and serine-supplemented piglets; C-D, results of ileum in control piglets and

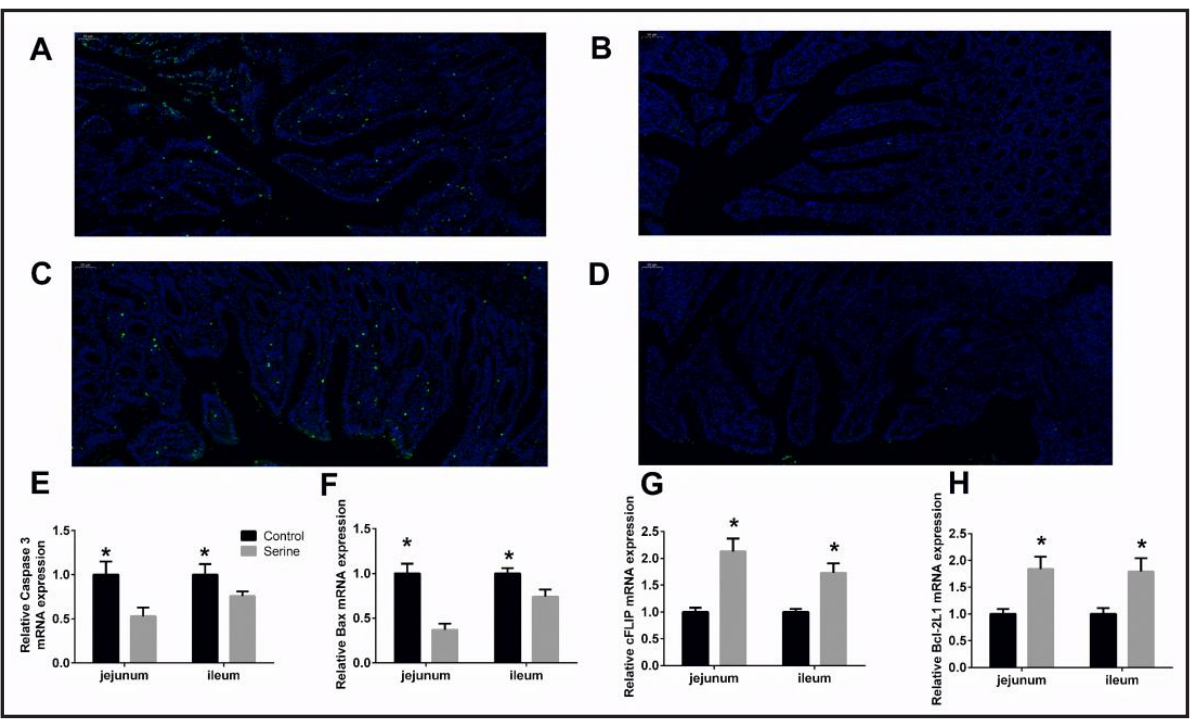

serine-supplemented piglets). Nuclei were stained with Hoechst 33342 (blue). E-H, relative mRNA expression of caspase 8, Bax, cFLIP and Bcl-2L1. Values are expressed as mean \pm SEM, $n=7 ;{ }^{*}<<0.05$.

\section{KARGER}


Fig. 5. Effects of serine on intestinal oxidative status in early-weaned piglets. CAT, catalase; GSH-Px, glutathione peroxidase; MDA, malondialdehyde; SOD, superoxide dismutase; Values are expressed as mean $\pm \mathrm{SEM}, \mathrm{n}=7$; $* \mathrm{p}<0.05$.
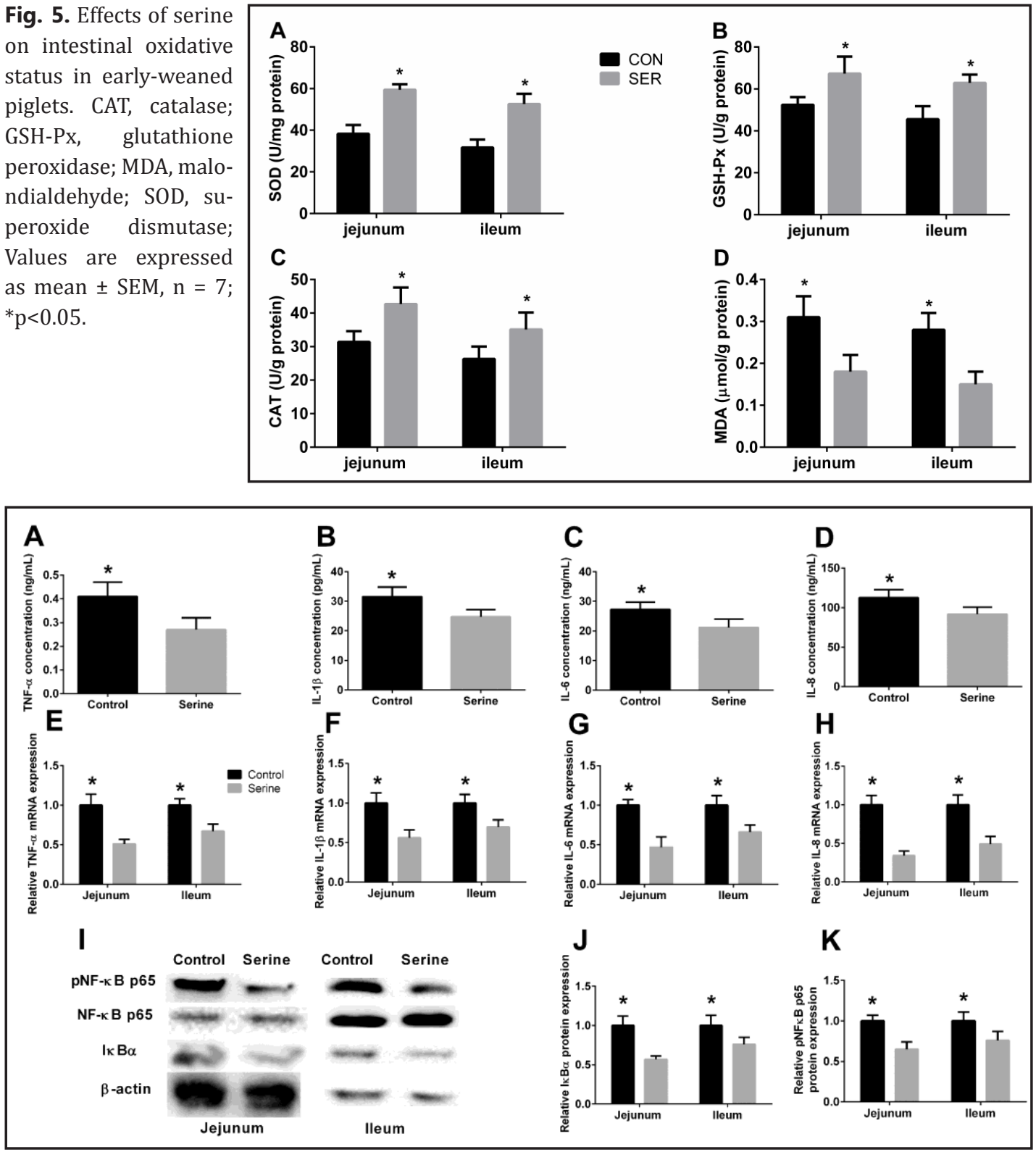

Fig. 6. Effects of serine on intestinal inflammation in early-weaned piglets. A-D, concentration of inflammatory cytokines in serum; E-H, mRNA of inflammatory cytokines in intestine; I-K, protein expression of

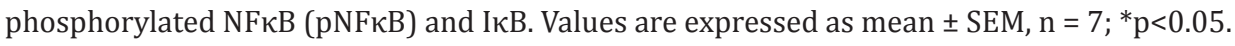

\section{Discussion}

Our results showed that piglets supplemented with serine had normal intestinal morphology, higher expression of tight junction proteins, and lower concentration or expression of markers of inflammation, apoptosis and oxidative stress, suggesting that serine improved intestinal health in early-weaned piglets. The improvement in daily body weight gain and diarrhea score may be a result of the beneficial effects of serine for maintaining gut health [12].

The small intestine is the major site of nutrient digestion and absorption in mammals. Villus height is an indirect indicator of the functional ability of intestinal epithelium cells as longer villi provide a larger absorptive area in the small intestine $[13,14]$. Our results showed that serine supplementation increased villus height in both the jejunum and ileum of piglets, 
suggesting that serine may be beneficial for epithelial differentiation. Weaning usually leads to villus atrophy caused by increased apoptosis and failure of enterocyte regeneration [13]. In the present study, we found higher levels of apoptosis and expression of pro-apoptotic genes in the epithelial villi of the jejunum and ileum of piglets in the Control group than in those in the Serine group. In association with these characteristics, both partial loss of villi and irregularly arranged microvilli were observed in Control piglets. In contrast, we did not find such unusual changes in piglets supplemented with serine. These results suggest that serine may help with maintaining gut health in weanling piglets.

Tight junctions are important apical intercellular structures in intestinal epithelial cells, and consist of cytosolic (e.g., ZO-1) and transmembrane (claudins and occludins) proteins. These proteins are critical for maintaining intestinal integrity. Normally, weaning causes decreases in tight junction protein expression, indicating the disturbances in the epithelial barrier [15]. We found that piglets supplemented with serine had higher expression of claudin-1, occludin and ZO-1, suggesting that serine may improve the building of tight junctions in the intestine of weanlings.

For further elucidation of the mechanisms involved in beneficial effects of serine on intestinal integrity, we determined the inflammatory response and oxidative status of the small intestine of piglets. In piglets, weaning leads to intestinal inflammatory responses that induce intestinal dysfunction $[16,17]$. Overexpression of cytokines plays a critical role in initiating inflammatory and pathological responses [16]. In the present study, concentrations of TNF- $\alpha$, IL-1 $\beta$, IL- 6 and IL-8 in serum, and their expression in the small intestine were both lower in serine-supplemented piglets than in Control piglets. These results are in line with our previous results for LPS-treated mice [8]. NF- $\kappa \mathrm{B}$ is activated in response to inflammation and the major signaling pathway modulates the expression of downstream cytokines involved in inflammatory response $[18,19]$. IкB is the key regulator of NF- $\kappa B[20]$. As expected, phosphorylation of NF- $\kappa$ B p65 and IкB was lower in serine-supplemented piglets than in Control piglets, suggesting lower activation of inflammation. The inflammatory response is always accompanied by oxidative stress in weanling piglets [2]. Reactive oxygen species cause tissue damage during the inflammatory response [21]. Previous studies demonstrated antioxidative effects of serine supplementation as they found that serine improved expression of glutathione peroxidase [22] and enhanced glutathione content [23]. Low concentration of MDA, and high concentration of SOD, GSH-Px, and CAT in both the jejunum and ileum indicate that serine may increase the anti-oxidative ability of the small intestine in earlyweaned piglets.

\section{Conclusion}

Our results show that dietary serine supplementation improves body weight gain, diarrhea score, and intestinal health by alleviating inflammatory responses and oxidative stress in early-weaned piglets. This is the first report demonstrating the beneficial effects of serine in piglets and our findings suggest that serine has the potential for use as a feed additive to prevent gut dysfunction caused by weaning. However, the underlying molecular mechanism for regulation of intestinal integrity and function by serine remains to be further elucidated.

\section{Abbreviations}

CAT (catalase); GSH-Px (glutathione peroxidase); $\mathrm{I} \kappa \mathrm{B} \alpha$ (inhibitor of nuclear factor kappa-B kinase-alpha); MDA (malondialdehyde); NF- $\mathrm{BB}$ (nuclear transcription factor kappa B); SOD (superoxide dismutase); TJPs (tight junction proteins). 


\section{Cellular Physiology Cell Physiol Biochem 2018;48:993-1002 \begin{tabular}{l|l} 
and Biochemistry Published 10.1159/000491967 & $\begin{array}{l}\text { (c) } 2018 \text { The Author(s). Published by S. Karger AG, Basel } \\
\text { www.karger.com/cpb }\end{array}$
\end{tabular}}

Zhou et al.: Serine Protects Intestinal Function in Piglet

\section{Acknowledgements}

This work was financially supported by National Natural Science Foundation of China (31702125; 31772642; 81273990), National Key Research and Development Program of China (2018YFD0500405; 2016YFD0501201; 2016YFD0500504), Agricultural Innovation Project of Hunan Province 2017YC03), Natural Science Foundation of Hunan Province (2017JJ3373) and the earmarked fund for China Agriculture Research System (CARS-35).

\section{Disclosure Statement}

The authors declare no competing financial interest.

\section{References}

1 Zhang Y, Zheng P, Yu B, He J, Yu J, Mao XB, Wang JX, Luo JQ Huang ZQ, Cheng GX, Chen DW: Dietary spraydried chicken plasma improves intestinal barrier function and modulates immune status in weaning piglets. J Anim Sci 2016;94:173-184.

2 Xu J, Xu C, Chen X, Cai X, Yang S, Sheng Y, Wang T: Regulation of an antioxidant blend on intestinal redox status and major microbiota in early weaned piglets. Nutrition 2014;30:584-589.

-3 Smith F, Clark JE, Overman BL, Tozel CC, Huang JH, Rivier JE, Blikslager AT, Moeser AJ: Early weaning stress impairs development of mucosal barrier function in the porcine intestine. Am J Physiol Gastrointest Liver Physiol 2010;298:G352-363.

4 Metcalf JS, Dunlop RA, Powell JT, Banack SA, Cox PA: L-Serine: a Naturally-Occurring Amino Acid with Therapeutic Potential. Neurotox Res 2018;33:213-221.

5 Hunter T: Why nature chose phosphate to modify proteins. Philos Trans R Soc Lond B Biol Sci 2012;367:2513-2516.

-6 Zhou X, He L, Wu C, Zhang Y, Wu X, Yin Y: Serine alleviates oxidative stress via supporting glutathione synthesis and methionine cycle in mice. Mol Nutr Food Res 2017;61.

7 Zhou X, He L, Zuo S, Zhang Y, Wan D, Long C, Huang P, Wu X, Wu C, Liu G, Yin Y: Serine prevented highfat diet-induced oxidative stress by activating AMPK and epigenetically modulating the expression of glutathione synthesis-related genes. Biochim Biophys Acta 2017;1864:488-498.

8 Zhou X, Zhang Y, He L, Wan D, Liu G, Wu X, Yin YL: Serine prevents LPS-induced intestinal inflammation and barrier damage via p53-dependent glutathione synthesis and AMPK activation. J Funct Foods 2017;39:225232.

9 Sweet LA, Kormegay ET, D. LM: The effects of dietary Luprosil NC on the growth performance and scouring index of weanling pigs. Agribiol Res 1990;43:271-282.

10 He L, Yang H, Hou Y, Li T, Fang J, Zhou X, Yin Y, Wu L, Nyachoti M, Wu G: Effects of dietary L-lysine intake on the intestinal mucosa and expression of CAT genes in weaned piglets. Amino Acids 2013;45:383-391.

-11 He L, Li H, Huang N, Tian J, Liu Z, Zhou X, Yao K, Li T, Yin Y: Effects of Alpha-Ketoglutarate on Glutamine Metabolism in Piglet Enterocytes in vivo and in vitro. J Agric Food Chem 2016;64:2668-2673.

12 He L, Huang N, Li H, Tian J, Zhou X, Li T, Yao K, Wu G, Yin Y: AMPK/alpha-Ketoglutarate Axis Regulates Intestinal Water and Ion Homeostasis in Young Pigs. J Agric Food Chem 2017;65:2287-2298.

13 Hampson DJ: Alterations in piglet small intestinal structure at weaning. Res Vet Sci 1986;40:32-40.

14 He L, Zhou X, Huang N, Li H, Cui Z, Tian J, Jiang Q Liu S, Wu J, Li T, Yao K, Yin Y: Administration of alphaketoglutarate improves epithelial restitution under stress injury in early-weaning piglets. Oncotarget 2017;8:91965-91978.

15 Hu CH, Xiao K, Luan ZS, Song J: Early weaning increases intestinal permeability, alters expression of cytokine and tight junction proteins, and activates mitogen-activated protein kinases in pigs. J Anim Sci 2013;91:1094-1101.

16 Al-Sadi R, Boivin M, Ma T: Mechanism of cytokine modulation of epithelial tight junction barrier. Front Biosci (Landmark Ed) 2009;14:2765-2778. 


\section{Cellular Physiology Cell Physiol Biochem 2018;48:993-1002 \begin{tabular}{l|l} 
DOI: 10.1159/000491967 & $\begin{array}{l}\text { O 2018 The Author(s). Published by S. Karger AG, Basel } \\
\text { www.karger.com/cpb }\end{array}$ \\
\hline
\end{tabular} \\ Zhou et al.: Serine Protects Intestinal Function in Piglet}

17 Pie S, Lalles JP, Blazy F, Laffitte J, Seve B, Oswald IP: Weaning is associated with an upregulation of expression of inflammatory cytokines in the intestine of piglets. J Nutr 2004;134:641-647.

18 Tak PP, Firestein GS: NF-kappaB: a key role in inflammatory diseases. J Clin Invest 2001;107:7-11.

19 Huang B, Xiao D, Tan B, Xiao H, Wang J, Yin J, Duan J, Huang R, Yang C, Yin Y: Chitosan Oligosaccharide Reduces Intestinal Inflammation That Involves Calcium-Sensing Receptor (CaSR) Activation in Lipopolysaccharide (LPS)-Challenged Piglets. J Agric Food Chem 2016;64:245-252.

-20 Goossens P, Vergouwe MN, Gijbels MJ, Curfs DM, van Woezik JH, Hoeksema MA, Xanthoulea S, Leenen PJ, Rupec RA, Hofker MH, de Winther MP: Myeloid IkappaBalpha deficiency promotes atherogenesis by enhancing leukocyte recruitment to the plaques. PLoS One 2011;6:e22327.

-21 Sido B, Hack V, Hochlehnert A, Lipps H, Herfarth C, Droge W: Impairment of intestinal glutathione synthesis in patients with inflammatory bowel disease. Gut 1998;42:485-492.

22 Wang Q, Sun LC, Liu YQ, Lu JX, Han F, Huang ZW: The Synergistic Effect of Serine with Selenocompounds on the Expression of SelP and GPx in HepG2 Cells. Biol Trace Elem Res 2016;173:291-296.

23 Sim WC, Yin HQ, Choi HS, Choi YJ, Kwak HC, Kim SK, Lee BH: L-serine supplementation attenuates alcoholic fatty liver by enhancing homocysteine metabolism in mice and rats. J Nutr 2015;145:260-267. 